\title{
Efficient wavefield inversion with outer iterations and total variation constraint
}

\author{
Chao Song, Tariq Alkhalifah
}

\begin{abstract}
Full-waveform inversion (FWI) is popularly used to retrieve a high-resolution velocity model that maximizes the data fitting directly. It is a highly non-linear optimization problem, and thus, FWI can fall easily into a local minimum. Wavefield reconstruction inversion (WRI) allows us to relax the wave equation constraint to provide a larger search space. It, however, requires high computational cost to update the velocity in each selected frequency through many expensive iterations. By recasting a linear optimization problem in terms of a modified source function (which includes the original source and secondary sources) and relying on the background velocity model, we end up with cheap inner iterations for inverting for the wavefield. We refer to this setup as efficient wavefield inversion (EWI). However, like WRI, EWI cannot mitigate the cycle-skipping problem completely when the background velocity model is far from the true one and low-frequency components in the data are missing. In this case, we propose to use additional outer iterations to better recover the velocity model. In the salt body inversion, we utilize a total variation (TV) regularization to constrain the inverted velocity model at each outer iteration. We demonstrate these features on a modified Marmousi model and a central part of the BP salt model. Application on a 2D real dataset also demonstrates the effectiveness of the proposed method.
\end{abstract}

Index Terms-Efficient wavefield inversion (EWI), cycleskipping, total variation constraint, wavefield reconstruction, frequency-domain.

\section{INTRODUCTION}

$\mathbf{F}$ ULL-waveform inversion (FWI) has become an effective technique to estimate high-resolution subsurface parameters. It takes advantage of all the information in the data, including diving waves, primary reflection waves, and multiples [1]. FWI heavily relies on the availability of long-offsets and diving waves in the data to recover the low wavenumber information. When the low wavenumber information matures after the early stages of FWI, reflection waves are used to introduce high wavenumber components to the model. The intermediate wavenumber gap due to the conventional surface seismic data insensitivity to it can be recovered by additional regularization terms and multiscattering illumination to the model [2]. In conventional FWI, the objective function based on the $l_{2}$ norm least-squares measurement is not convex [3]. We can easily fall into local minima when we use gradient-based methods to optimize this problem. The success of FWI depends on the availability of low-frequency information and diving waves in the data to reconstruct the low-wavenumber part of the model early in the inversion process. In reality, due to practical limits in acquisition, low-frequency and diving wave information is

The authors are with the Physical Sciences and Engineering Division, King Abdullah University of Science and Technology (KAUST), Saudi Arabia, 23955 (e-mail:chao.song@ kaust.edu.sa;tariq.alkhalifah@kaust.edu.sa). often unavailable in the dataset. In order to obtain reasonable inverted models, we often pursue a good initial model for FWI, such as using traveltime tomography [4], [5], wave equation based traveltime inversion [6], [7], migration velocity analysis (MVA) methods [8], [9], Laplace-domain waveform inversion [10], and seismic envelop inversion [11], [12], [13]. These methods extract specific features from the seismic data to confirm the convexity of the objective function. Reflectionwaveform inversion (RWI) was developed to access the smooth update information along the reflection ray wavepath [14], [15]. After reconstruting the low-wavenumber components of the model, a sequential FWI implementation is able to generate a high-resolution inverted result. The original RWI implementation requires a well-calculated true-amplitude reflectivity using the least-squares optimization and a sequential FWI implementation, so this process is expensive [16]. To improve the computational efficiency, a simultaneous inversion for the background model and perturbations using a new objective function was proposed [17]. Proper gradient filtering methods considering the relation between the scattering angle and model wavenumber can also help mitigate the cycle-skipping issue [18], [19], [20].

Besides approaches used to generate a good initial model, new objective functions immune to the cycle-skipping problem were also introduced. Adaptive waveform inversion introduced a matching filter which aims at forcing a zero-lag delta function instead of comparing data pixel by pixel [21], [22]. A proper regularization terms will also be helpful to produce smooth inversion result, like Tikhonov regularization [23]. However, Tikhonov regularization tends to hurt sharp interfaces corresponding to large-contrast regions. Total variation (TV) regularization was proposed to preserve sharp edges for salt body inversion [24]. To further improve the performance of regularization, compound regularization was used to combine the advantages of Tikhonov regularization and TV regularization [25].

Wavefield reconstruction inversion (WRI) was proposed to use the wave equation as an additional regularization term, instead of a hard constraint. This allows for a larger searchspace over both the wavefields and the model [26], [27]. By extending the source function beyond a point source, reconstructed wavefields are able to fit the recorded data better than the original WRI [28], [29], [30]. In WRI (or modified WRI), the velocity is updated at every iteration, which consequently means an updated wave equation operator. As a result, many expensive iterations in each frequency are required, as the model is updated at every iteration.

To further improve the computational efficiency, an effi- 
cient wavefield inversion (EWI) was proposed [31]. They specifically recast the wavefield optimization with respect to a background model and a modified source and calculate the velocity perturbations using direct division (deconvolution) [32]. By using a modified source function as an independent parameter to capture velocity perturbations, it allowes us to include multiscattering components in the velocity update. In this case, we end up with cheap inner iterations (no update in the background operator) in updating the wavefield and the modified source function. Using direct division, the velocity perturbation is calculated. They implemented this method in the frequency domain, and in each selected frequency, the background operator remains stationary. Although EWI is reliable and efficient, it cannot mitigate the cycle-skipping problem completely when the required velocity perturbation is large. Under this condition, using the outer iterations to update the velocity model throughout the whole selected frequency band can gradually improve the inversion result. For the salt body inversion, total variation (TV) is used to constrain the inverted velocity to remove the high oscillatory artifacts and preserve the edges [33], [34]. We utilize it here, as well.

In this paper, we first review the theory of EWI, and explain why inner iterations between the wavefield and a modified source function allows us to include multiscattering signals in the reconstructed wavefield theoretically. However, EWI is not powerful enough to completely mitigate the cycleskipping issue when the velocity perturbation is large and lowfrequency signals in the data are missing. Thus, we add outer iterations to the EWI process to improve the accuracy of the inversion. For the salt body inversion, we use a TV constraint to remove the undesired artifacts and preserve the sharp edges of a salt body. We test the proposed method from a frequency of $3 \mathrm{~Hz}$. Finally, we, also, apply this method on real data.

\section{METHOD}

In this section, we first explain the theory of the efficient wavefield inversion and the total variation constraint. Then, we use a simple anomaly example to show the importance of the outer iterations.

\section{A. Efficient wavefield inversion}

In the conventional FWI implementation, we tend to measure the misfit between recorded and predicted data by constraining the wave equation, as follows:

$$
\min J(V)=\min \sum_{i} \frac{1}{2}\left\|d_{i}-C u_{i}\right\|_{2}^{2} \quad \text { s.t. } \quad L(V) u_{i}=f_{i},
$$

where, $\|\cdot\|_{2}^{2}$ denotes the $l_{2}$ norm, and $i$ denotes the source index. $C$ is the mapping operator, which projects the wavefield $u$ to the receiver positions. $L(V)=\left(V_{0}+V\right) \omega^{2}+\nabla^{2}=$ $L_{0}+\omega^{2} V$ is the modelling operator, with the operator $L_{0}=$ $V_{0} \omega^{2}+\nabla^{2}$ corresponding to the background squared slowness $V_{0} . f_{i}$ represents the original source function. $V$ corresponds to, in this case, the unknown squared slowness perturbation, and we set it as zero at the beginning of the inversion process. The squared slowness gradient in FWI is given by:

$$
\nabla_{V} J=\sum_{i} \omega^{2} u_{i} L^{-T}\left(C^{T} \Delta d_{i}\right)
$$

where $\Delta d_{i}=d_{i}-C u_{i}$ is the data residual, and $L^{-T}\left(C^{T} \Delta d_{i}\right)$ denotes the back-propagated wavefield. $\nabla_{V} J$ is given by elements that span the model space. The background wavefield $u$ is calculated using the wave equation, which depends on the velocity. This dependency causes the non-linearity of the objective function, which results in cycle-skipping issue in FWI. In order to solve this problem, WRI was proposed and its objective function is given by [26]:

$$
\min E\left(V, u_{i}\right)=\min \frac{1}{2} \sum_{i}\left\|d_{i}-C u_{i}\right\|_{2}^{2}+\frac{\alpha^{2}}{2} \sum_{i}\left\|L(V) u_{i}-f_{i}\right\|_{2}^{2} .
$$

In this formulation, the wave equation is added as an additional regularization constrain term, scaled by $\alpha^{2}$. A proper $\alpha^{2}$ can be found by scaling the highest eigenvalue of the augmented linear system [27]. The weight $\alpha^{2}$ can also be automatically selected by using a multiplicative cost function [35]. A discrepancy-based method was also proposed to control the penalty parameter [36]. The sensitivity of the EWI objective function with respect to $\alpha^{2}$ is discussed in [37]. Following the perturbation theory, we split the velocity model into the background velocity and a perturbation, so the wave equation is formulated as:

$$
L u=f \rightarrow\left(L_{0}+\omega^{2} V\right) u=f \rightarrow L_{0} u=f_{e},
$$

where, $f_{e i}=f_{i}-\omega^{2} V u_{i}$ represents a modified source function. Now we can present an optimization for the wavefield independent of the velocity perturbation in the wave equation operator, given by:

$\min E\left(u_{i}, f_{e i}\right)=\min \frac{1}{2} \sum_{i}\left\|d_{i}-C u_{i}\right\|_{2}^{2}+\sum_{i} \frac{\alpha^{2}}{2}\left\|L_{0} u_{i}-f_{e i}\right\|_{2}^{2}$.

In equation 5 , there are two independent variables: the wavefield $u_{i}$ and the modified source function $f_{e i}$. The wavefield satisfies the following linear equation:

$$
\left(\begin{array}{c}
\alpha L_{0} \\
C
\end{array}\right) u_{i}=\left(\begin{array}{c}
\alpha f_{e i} \\
d_{i}
\end{array}\right)
$$

In the implementation of solving the wavefield, we keep the operator $L_{0}$ stationary for each frequency. In the beginning of inversion process, we set the squared slowness perturbation $V$ to zero, and $f_{e i}=f_{i}$, which is the true source. The objective function $E$ is a quadratic function of the modified source function $f_{e i}$, and it can be evaluated using:

$$
f_{e i}=L_{0} u_{i}
$$

We use inner iterations between equations 6 and 7. From the linear equation 6 , the wavefield is expected to have initially only single scattering components from the unknown perturbations and the secondary sources (medium parameter perturbations) stored in $f_{e}$. Inner iteration adds the scattering components to the wavefield. As the background operator $L_{0}$ remains the same during the inner iterations, no inversions of the operator in equation 6 is required. After obtaining an accurate wavefield $u$ and a modified source function $f_{e i}$, 
the squared slowness perturbation can be calculated by a deconvolution process:

$$
V=\sum_{i} \frac{\left(f_{i}-f_{e i}\right)}{\omega^{2} u_{i}} \approx \sum_{i} \frac{\left(f_{i}-f_{e i}\right) u_{i}^{*}}{\omega^{2} u_{i} u_{i}^{*}+\lambda},
$$

where $\lambda$ is a small value to avoid the potential singularity in the inverted results. Usually, we use one percentage of the maximum value of the wavefield amplitude $u^{*} u$. This deconvolution process in equation 8 makes $V$ an approximate solution to the true perturbation. So we formulate an objective function $J(\gamma)=\frac{1}{2} \sum_{i}\left\|f_{e i}-f_{i}+\gamma \omega^{2} V u_{i}\right\|_{2}^{2}$ to find a step length $\gamma$ to optimize the update. As $J(\gamma)$ is a function of only $\gamma$, we use a global optimization method to calculate $\gamma$ [38], and the cost is negligible. Finally, the background model is evaluated using:

$$
V_{0}=V_{0}+\gamma V \text {. }
$$

The proposed method is capable of calculating the velocity perturbation including multiscattering components directly in each frequency, so the convergence rate for the inversion accelerates [39], [40]. However, if the initial velocity model is far from the true one, we propose to use outer iterations to repeat the updating scheme over the full frequency band. Usually, only a limited number of outer iterations are required.

\section{B. Total variation constraint}

The inverted velocity model is sometimes contaminated by high wavenumber oscillations. Total variation (TV) regularization is a popularly used method to preserve the sharp edges without over smoothing the reconstructed model [34]. An iterative TV smoothing method is used to remove the high wavenumber oscillations in the inverted velocity model [41], [42]. Assuming that the inverted velocity model is shown as:

$$
V_{0}=V_{t v}+\epsilon,
$$

where $V_{t v}$ represents the desired clean velocity model after TV denoising, $\epsilon$ represents the undesired oscillations, and $V_{0}$ represents the originally updated background velocity model. We want to remove $\epsilon$ from $V_{0}$, and the minimization of the $\mathrm{TV}$-penalized least-squares objective function is given by:

$$
f\left(V_{t v}\right)=\frac{1}{2}\left\|V_{t v}-V_{0}\right\|_{2}^{2}+\beta J_{\mu}\left(V_{t v}\right),
$$

where

$$
J_{\mu}\left(V_{t v}\right)=\int_{\Omega} \sqrt{\left|\nabla V_{t v}\right|_{2}^{2}+\mu} d \mathbf{x} .
$$

In equation $12, \nabla V_{t v}=\left(\frac{\partial V_{t v}}{\partial x}, \frac{\partial V_{t v}}{\partial z}\right) . \mu$ is a small positive value, and $\beta$ controls the weight of the total variation. We use $g\left(V_{t v}\right)$ to denote the gradient with respect to $V_{t v}$ of the equation 11, and it is given by:

$$
g\left(V_{t v}\right)=V_{t v}-V_{0}+\beta \nabla \cdot\left(\frac{\nabla V_{t v}}{\sqrt{\left|\nabla V_{t v}\right|_{2}^{2}+\mu}}\right) .
$$

Finally, we use a gradient descent scheme to update $V_{t v}$, as given by:

$$
V_{t v}^{(k+1)}=V_{t v}^{(k)}-\tau_{k} g\left(V_{t v}^{(k)}\right), \quad k=0,1,2 \ldots
$$

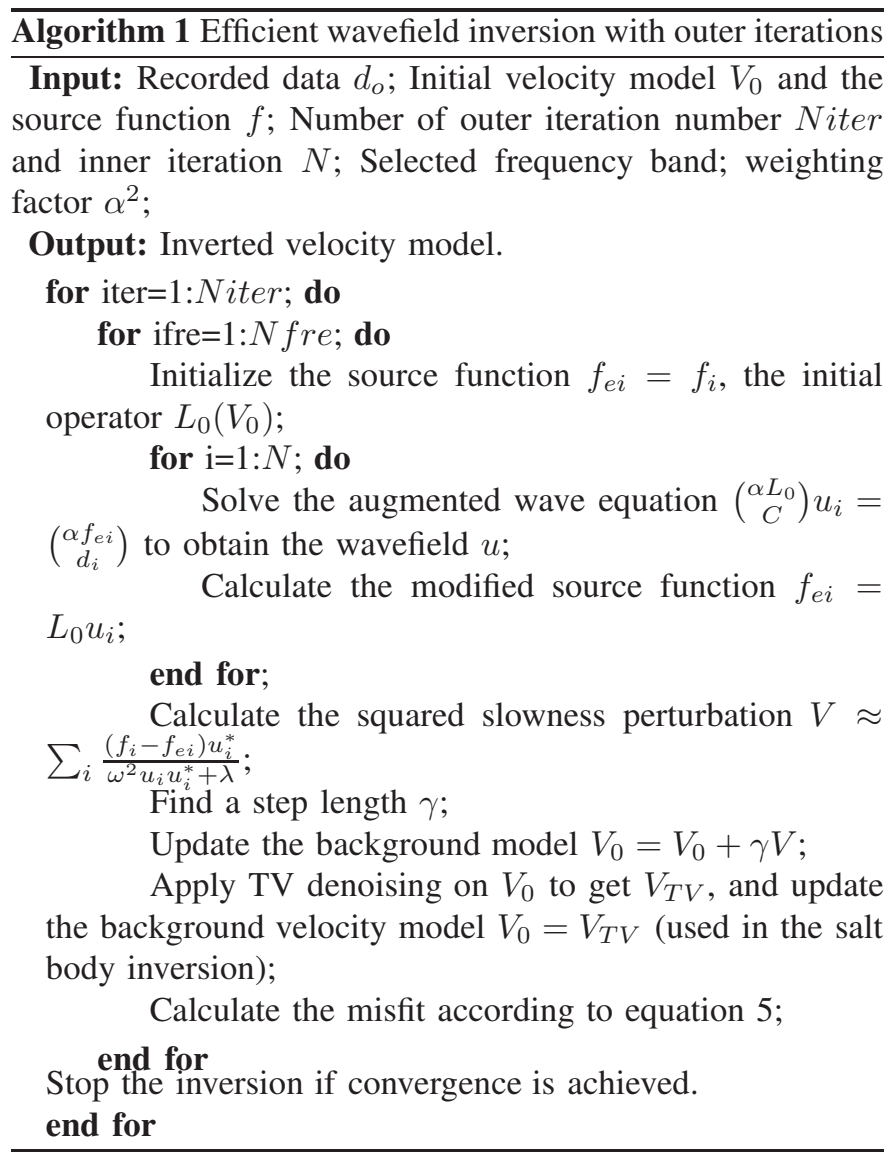

where $k$ is the $k$ th iteration, and $\tau_{k}$ is the step length. We use $\tau_{k}=0.2$ and $\beta=0.1$ in the salt body inversion shown in this paper, and they are extracted from trial and error. As this update scheme is a linear process, it will only add little extra cost. The detailed algorithm is explained in the Algorithm 1.

\section{Outer iterations}

Outer iterations in frequency domain waveform inversion implementation are sometimes used to further cement the data fitting for low frequencies after including updates from the high frequencies [43]. The outer iterations involve repeating EWI from low to high frequencies once again starting from the velocity model obtained in the previous EWI sweep. Since, in our EWI implementation, we update the velocity once per frequency, the outer iterations are expected to add additional information as the velocity model matures (Algorithm 1). The outer iteration also can be used to include more data in the inversion as we can include frequencies in the second sweep which are not used in the first sweep.

We initially test these features on a homogeneous background with two strong square anomalies in the model. The background homogeneous velocity is $2320 \mathrm{~m} / \mathrm{s}$, and the velocity of the anomalies are $3000 \mathrm{~m} / \mathrm{s}$ as shown in Fig. 1a. We set 20 sources on the surface, and receivers are placed on all the boundaries. The source function we use is ricker wavelet with dominant frequency being $8 \mathrm{~Hz}$. We use a narrow frequency band from $4 \mathrm{~Hz}$ to $6 \mathrm{~Hz}$ with a frequency interval of $0.5 \mathrm{~Hz}$ to conduct our inversion. We use two inner iterations in each 

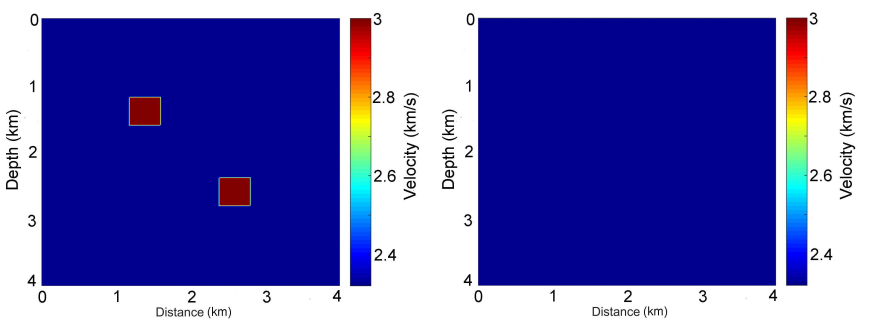

Fig. 1. The true scatter velocity model (a), and the true background velocity model (b).

frequency. The $\alpha^{2}$ we use in this paper for all the examples is $10 e^{7}$, which was chosen on a trial and error basis.

If we know the background velocity and use it (Fig. 1) as the initial velocity, the inverted velocity using EWI is shown in Fig. 2. We also perform FWI and WRI using the same inversion setup, and get the results as shown in Figs. 3a and $3 \mathrm{~b}$, respectively. It is obvious that EWI has a better recovery of the anomalies than FWI and WRI. We decrease the weighting factor $\alpha^{2}$ in WRI to enhance the data fitting. The inverted results for WRI with $\alpha^{2}=10 e^{5}, 10 e^{3}$ are shown in Figs. 3c and $3 \mathrm{~d}$, respectively. They show no obvious improvements, as the velocity perturbation is large and number of outer iterations are limited.

However, the background velocity is difficult to retrieve in reality. If we use a wrong background model of $2500 \mathrm{~m} / \mathrm{s}$ as the initial velocity (Fig. 4b), the inverted velocity models using EWI after one, two, three and four outer iterations are shown in Figs. 5a, 5b, 5c, and 5d, respectively. From the velocity update progress, we see that the background velocity is gradually corrected. After the first outer iteration, the strong square anomalies starts to show up. This can be explained from the difference between the modified source function and original source function summed over all the sources, as shown in Figs. 6a, 6b, 6c, and 6d, respectively. Fig. 6 shows that the source difference is large at the beginning of the inversion, and mainly focused on the background. The source difference gets weaker and weaker as the velocity accuracy improves. We show the data misfit curve and the wave equation misfit curve for the full frequency band at each outer iteration in Figs. 7a and $7 \mathrm{~b}$, respectively. They demonstrate that the convergence speed of EWI benefits a lot from the outer iterations. If we apply TV constraint to the inverted velocity in each frequency, the final inverted velocity using EWI is shown in Fig. 8. We observe that using TV constraint to the velocity, the artifactes in the shallow part are removed without hurting the sharp edges of the anomalies. The inverted velocity model with four iterations in each frequency (classic way) using FWI and WRI are shown in Figs. 9a and 9b, respectively. FWI and WRI fail to invert for a reasonable velocity model using such a limited iteration number.

\section{NUMERICAL TEST}

We will now share the results from implementing this approach on a modified Marmousi and the BP salt body models. Finally, we test the approach on field data from

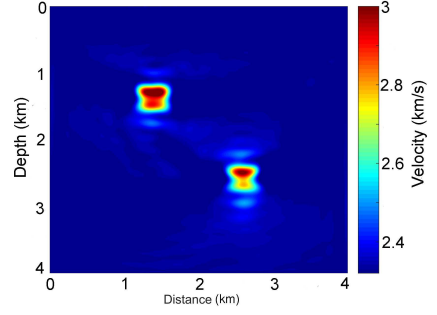

Fig. 2. The inverted velocity model after four iterations using EWI corresponding to the true background velocity.
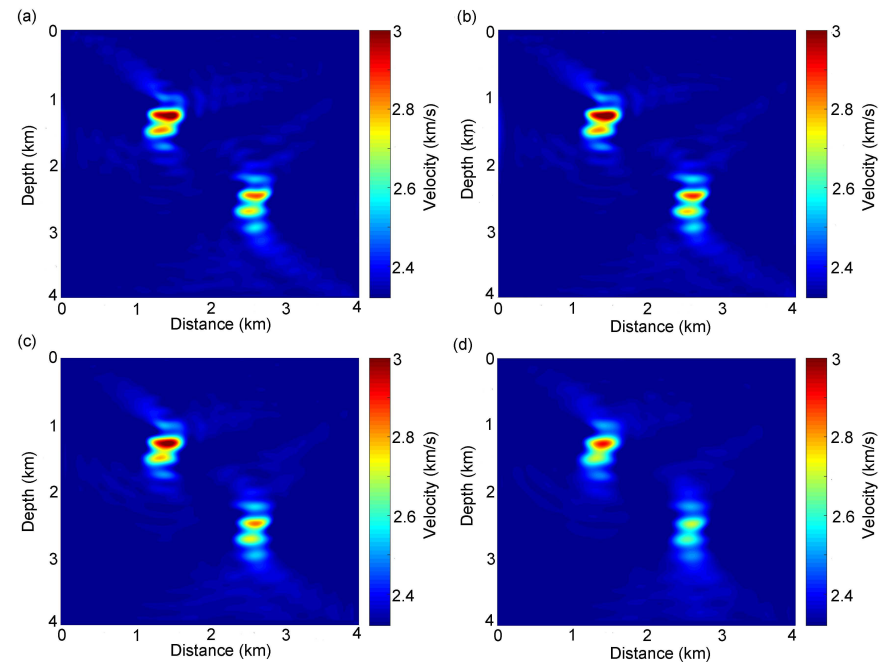

Fig. 3. The inverted velocity model after four iterations using FWI (a), WRI with $\alpha^{2}=10 e^{7}(\mathrm{~b}), \alpha^{2}=10 e^{5}(\mathrm{c})$, and $\alpha^{2}=10 e^{3}$ (d) corresponding to the true background velocity.

Western Australia, in which we also have check shot well information to compare with.

\section{A. 2D Marmousi model}

We first apply the proposed approach on a modified Marmousi model of size $370 \times 90$ samples. The true velocity model is shown in Fig. 10a. We use a linearly increasing velocity with depth as the initial velocity model, as shown in Fig. 10b. The horizontal and vertical grid sampling interval is $25 \mathrm{~m}$.

Since low frequencies in the data are often contaminated with noise, we start the inversion from $4 \mathrm{~Hz}$ up and stop at $10 \mathrm{~Hz}$ with a frequency interval of $0.5 \mathrm{~Hz}$. We use 18 sources uniformly distributed on the surface, and all the grid points
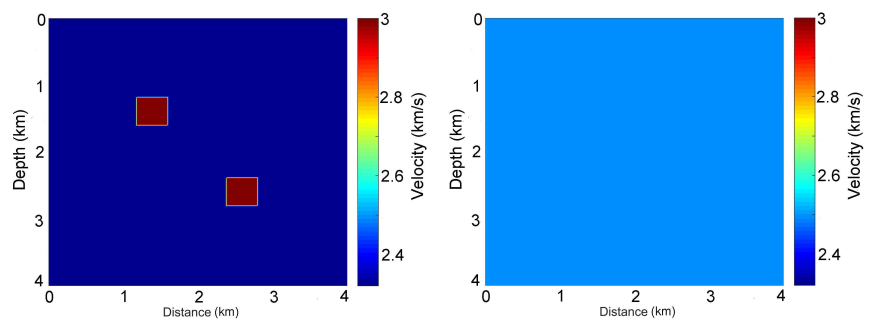

Fig. 4. The true scatter velocity model (a), and a wrong background velocity model (b). 

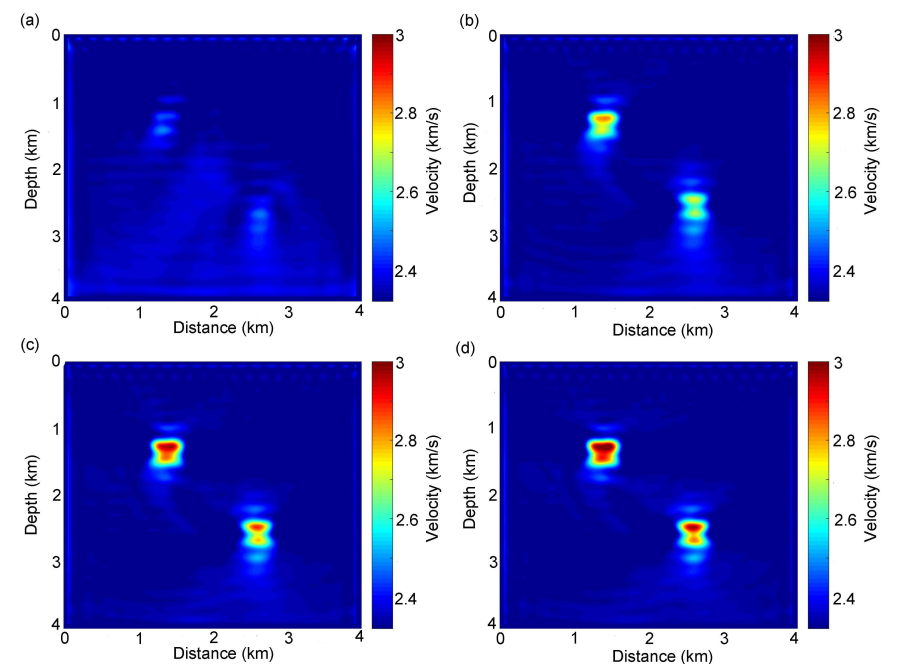

Fig. 5. The inverted velocity model after one (a), two (b), three (c), and four (d) outer iterations using EWI corresponding to the wrong background velocity.
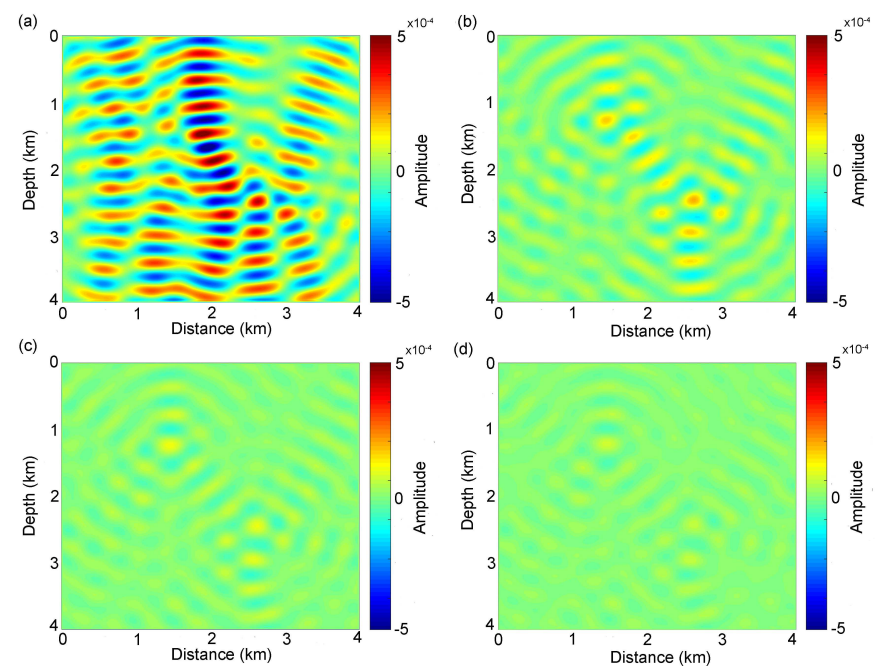

Fig. 6. The difference between the modified source function and original source function $\sum_{i} f_{e i}-f_{i}$ after one (a), two (b), three (c), and four (d) outer iterations.
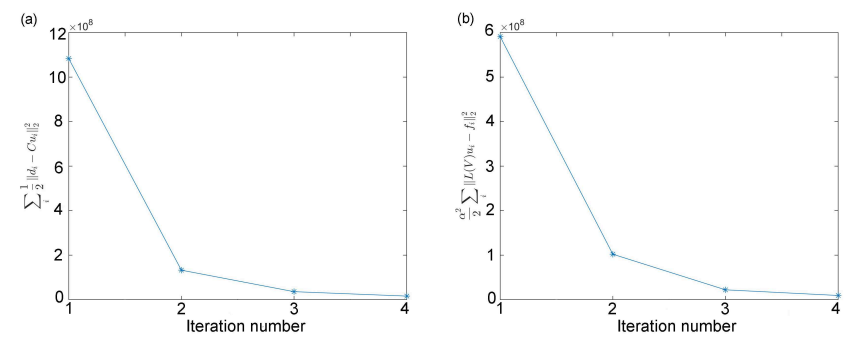

Fig. 7. The data misfit curve $\frac{1}{2} \sum_{i}\left\|d_{i}-C u_{i}\right\|_{2}^{2}$ (a), and wave equation misfit $\sum_{i} \frac{\alpha^{2}}{2}\left\|L_{0} u_{i}-f_{e i}\right\|_{2}^{2}$ curve (b) for EWI.

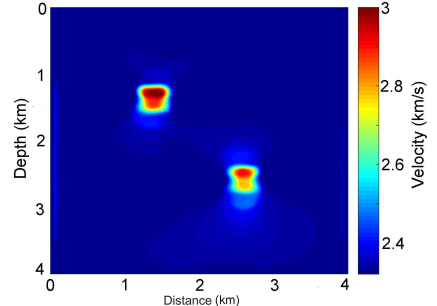

Fig. 8. The inverted velocity model after four iterations using TV constrained EWI corresponding to the wrong background velocity.
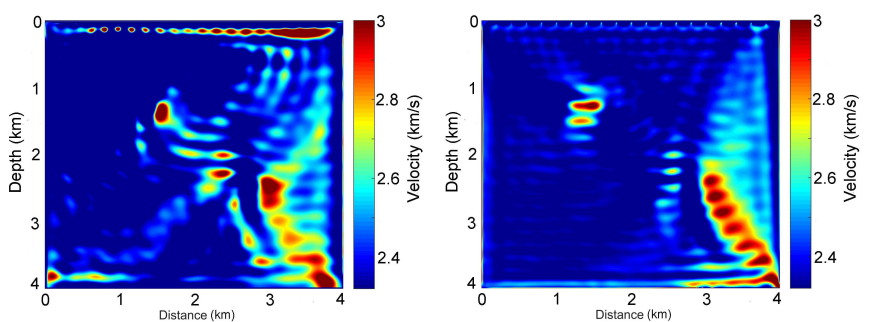

Fig. 9. The inverted velocity model after four iterations using FWI (a), and WRI (b) corresponding to the wrong background velocity.

on the surface act as receivers. We generate the synthetic data in the frequency domain using a delta function source. We use two inner iterations, considering higher-order scattering energy tends to be weak in this example. At $4 \mathrm{~Hz}$, we show in Fig. 11 how $J(\gamma)$ as a function of the step length $\gamma$. After the line search process, we end up with a step length $\gamma=0.59$. The inverted velocity model using EWI is shown in Fig. 12 without outer iterations. With almost the same computational cost (one iteration per frequency), the inverted velocity models using FWI and WRI are shown in Fig. 13a and $13 \mathrm{~b}$, respectively. EWI recovers most of the Marmousi model and contains less artifacts than FWI and WRI as shown in the circled areas in Figs. 12 and 13. We attribute this to the addition of inner iterations, and the use of direct division to compute the velocity perturbation. As we miss low-frequency information and use only one iteration per frequency, only the shallow part of the model is recovered well.

Next, we use five outer iterations, and thus, the inverted velocity model is shown in Fig. 14. It shows that after five outer iterations, we recover the velocity model even at depth well. Using the same number of iterations, the inverted velocity models using FWI and WRI are shown in Figs. 15a and 15b,
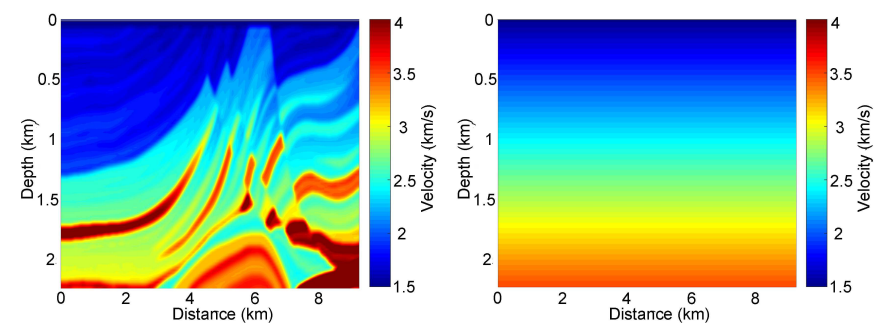

Fig. 10. The true modified Marmousi velocity (a), and the initial velocity (b). 


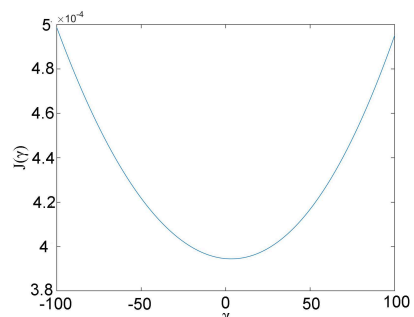

Fig. 11. Function $J(\gamma)$ of the step length $\gamma$.

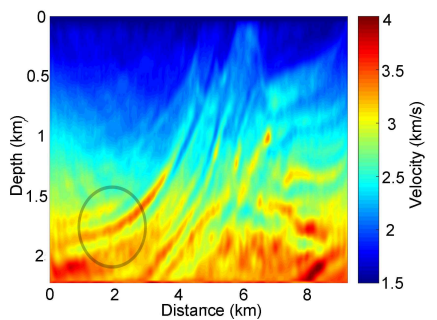

Fig. 12. The inverted velocity model using EWI with one iteration (without outer iteration).

respectively. It is obvious that FWI falls into a local minimum, and resulting model contains more artifacts. Although WRI improves the inversion result to some extent, the deep area in the model is still not well recovered. Vertical velocity profiles in Fig. 16 show that, the initial velocity (red line) is far away from the true velocity (black line). The inverted results from FWI (blue line) and WRI (pink line) clearly suffer from the cycle-skippig problem. Only the inverted velocity using EWI (green line) reasonably fits the true velocity.

The cost of data collection and waveform inversion will be dramatically reduced using a multi-source waveform inversion [44]. Conventional gradient-based FWI suffers from the cross-
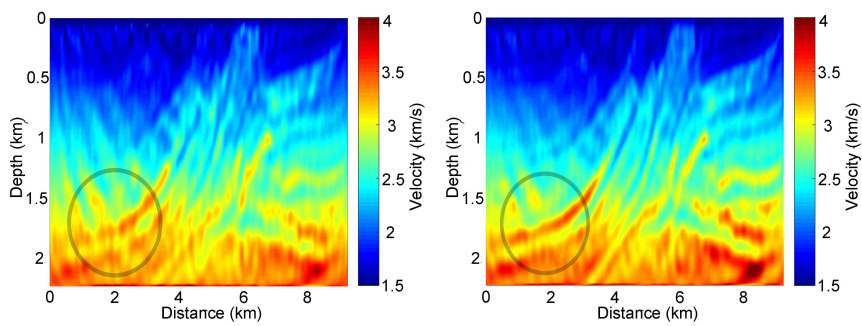

Fig. 13. The inverted velocity model using FWI (a) and WRI (b) with one iteration.

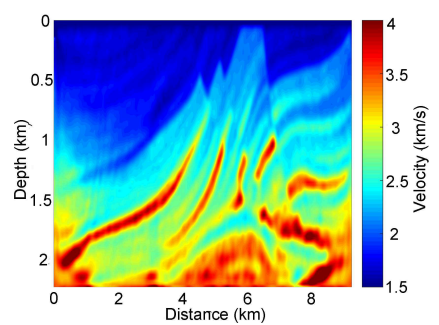

Fig. 14. The inverted velocity model using EWI after five outer iterations.
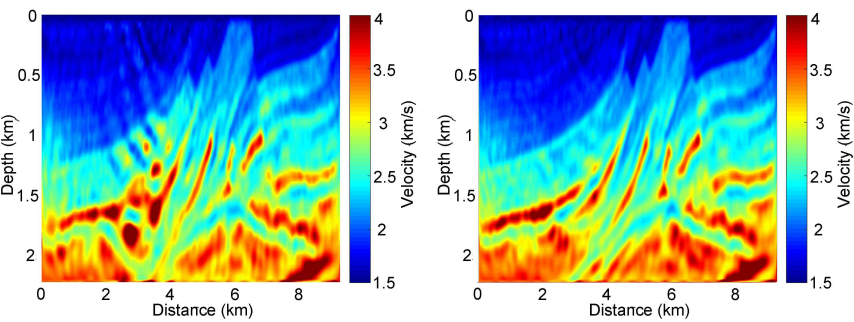

Fig. 15. The inverted velocity model using FWI (a) and WRI (b) after five iterations.
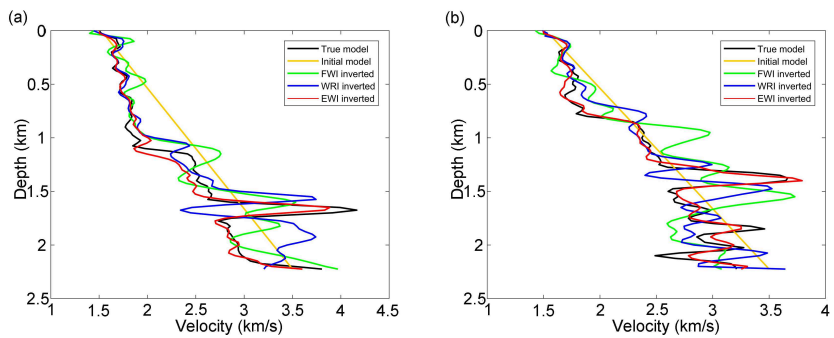

Fig. 16. The velocity profiles at $2.5 \mathrm{~km}$ (a) and $3.75 \mathrm{~km}$ (b).

talk artifacts due to cross correlation of unrelated events from the different sources. As EWI calculates the velocity perturbation using a direct division, it may reduce the crosstalk artifacts in a multi-source waveform inversion. With the same inversion setup and strategy, we fire three simultaneous sources randomly chosen at one time, and repeat this process six times to make sure all 18 sources are utilized. In this multisource waveform inversion, we also use 5 outer iterations. The inverted result using EWI is shown in Fig. 17, and the inverted results using FWI and WRI are shown in Fig. 18a and 18b, respectively. We observe that the inversion result from EWI includes far less the cross-talk artifacts than those results from FWI or WRI.

\section{B. 2004 BP salt model central target}

We now consider a more challenging application with a central target of the 2004 BP salt model with a modification in size to allow for efficient computation. The true model contains a large salt body in the center shown in Fig. 19. The size of this submodel is $300 \times 80$ grid points with $40 \mathrm{~m}$ spacing in both directions. The initial velocity model we use is a background velocity without any salt information, which is shown in Fig. 20.

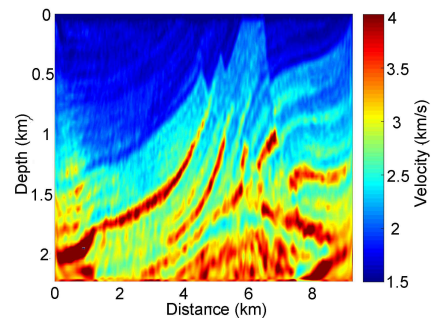

Fig. 17. The inverted velocity model using EWI in multi-source waveform inversion. 

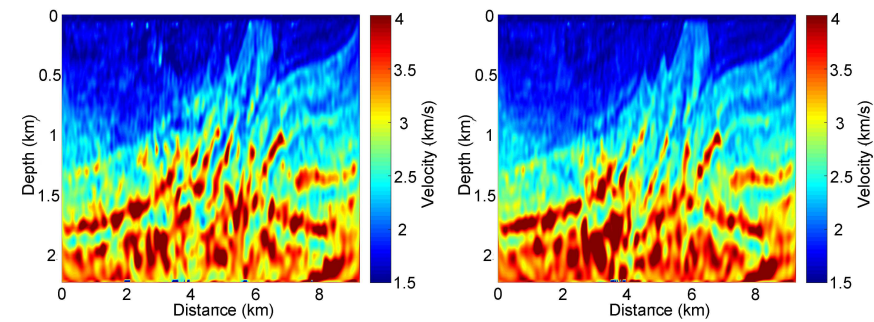

Fig. 18. The inverted velocity models using FWI method (a) and WRI (b) in multi-source waveform inversion.

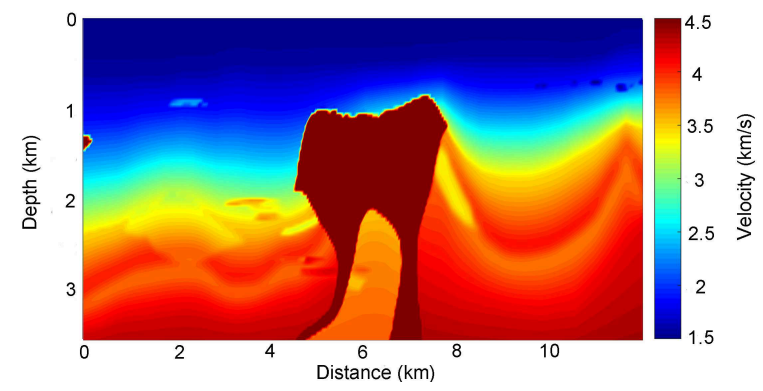

Fig. 19. The true 2004 BP salt model central target.

The selected frequency band is from $3 \mathrm{~Hz}$ to $10 \mathrm{~Hz}$ with a frequency sampling interval of $0.25 \mathrm{~Hz}$. We use 30 sources uniformly distributed on the surface, and all the grid points on the surface act as receivers. We still use a delta function source to generate the synthetic data in the frequency domain. We use two inner iterations per frequency, and 15 outer iterations to repeat the full spectrum inversion. In the salt body inversion, we first use EWI without TV constraint to perform the inversion. The inverted velocity models after 5 , 10, and 15 outer iterations are shown in Figs. 21a, 21b, and $21 \mathrm{c}$, respectively. We observe that after 5 outer iterations, the top part of the salt body is captured. However, there is an obvious gap between the top and the bottom of the salt body. With the increase of outer iterations, the true salt body structure is recovered. But there exist a lot of highwavenumber oscillations in the inverted model. To further improve the inverted velocity, we apply the TV constraint to the inverted velocity at each frequency. The inverted velocity models using EWI with TV constraint after 5, 10, and 15 outer iterations are shown in Figs. 22a, 22b, and 22c, respectively.

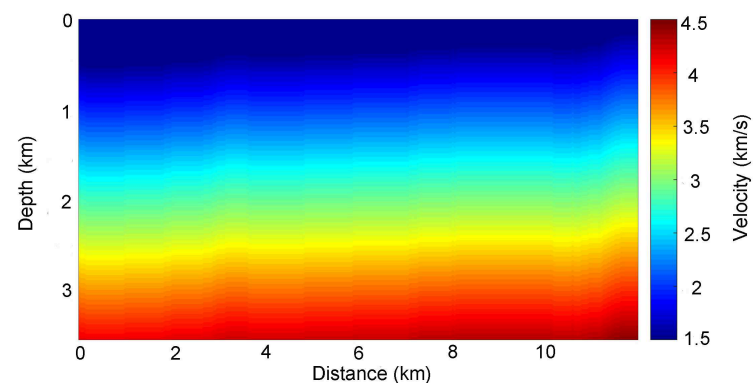

Fig. 20. The initial BP salt model.
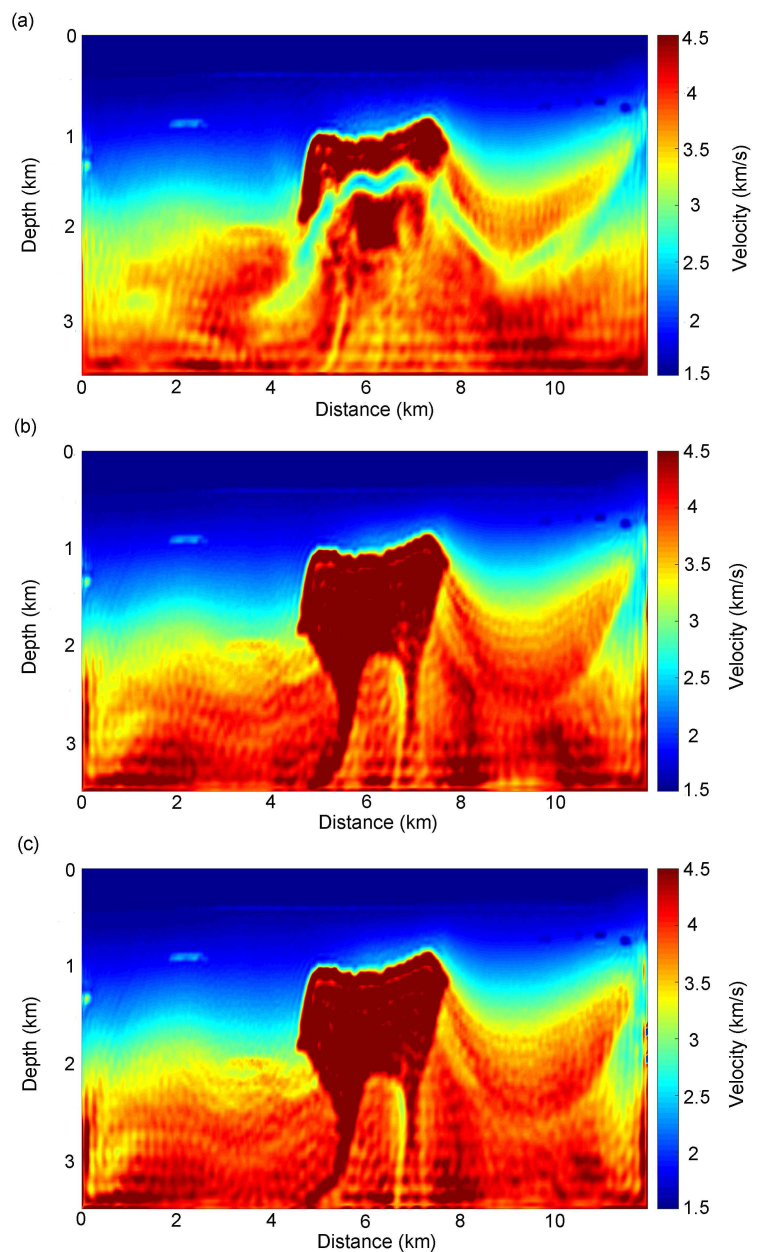

Fig. 21. The inverted BP salt model using EWI without TV constraint after 5 (a), 10 (b), and 15 (c) outer iterations.

With the help of the TV constraint, the high-wavenumber oscillatory artifacts are completely removed, while the sharp edges of the salt body are preserved. In the final inverted velocity model shown in Fig. 22c, it is obvious that the salt body in the center is perfectly reconstructed including the low velocity area beneath the salt. We show the percent root-meansquare (RMS) errors of the inverted models with and without TV constraint per outer iteration in Fig. 23. EWI without using TV constraint shows a reasonably good convergence, and TV constraint helps EWI converge to a lower model RMS error. To demonstrate the superiority of EWI, we implement the same inversion setup and strategy using WRI. The final inverted result using WRI is shown in Fig. 24. Fig. 24 shows obvious cycle skipping, and this problem comes from the limited number of iterations in the low frequency. The advantage of TV constrained EWI in the salt body inversion is shown more clearly in a velocity profile at $6 \mathrm{~km}$, as shown in Fig. 25. It is obvious that the inverted velocity model using TV constrained EWI agrees with the true velocity model reasonably well.

\section{2 D real data}

The 2D real data we use is acquired by CGG using a Broadseis acquisition system from North-Western Australia 

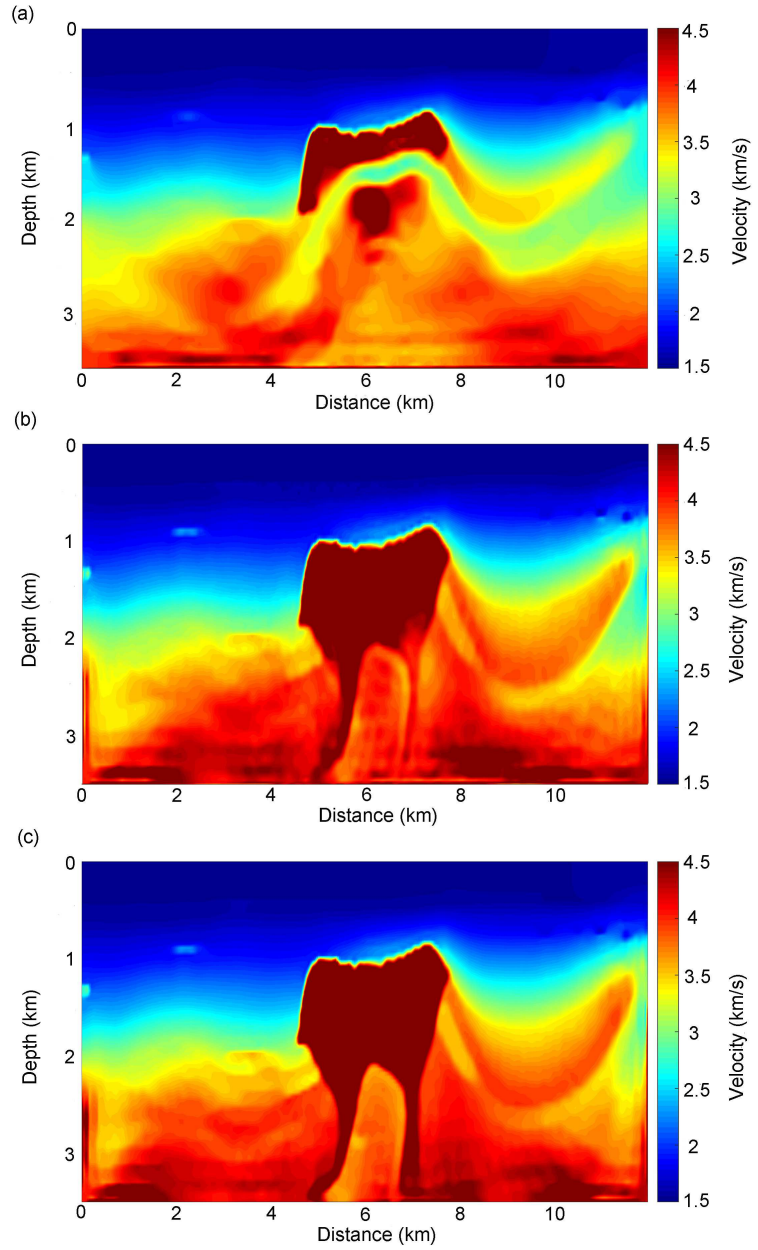

Fig. 22. The inverted BP salt model using EWI with TV constraint after 5 (a), 10 (b), and 15 (c) outer iterations.

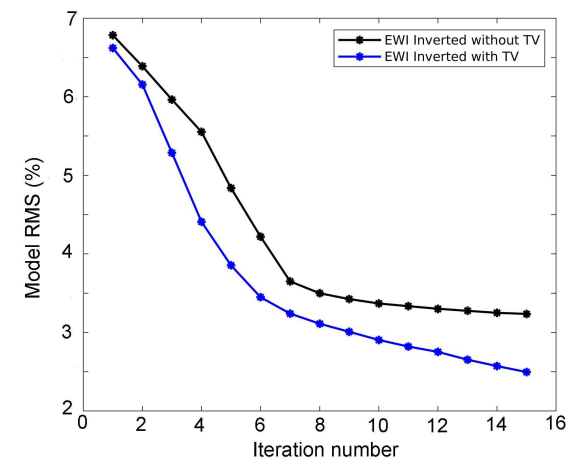

Fig. 23. The percent root-mean-square (RMS) error of the inverted models with (blue line) and without (black line) TV constraint.

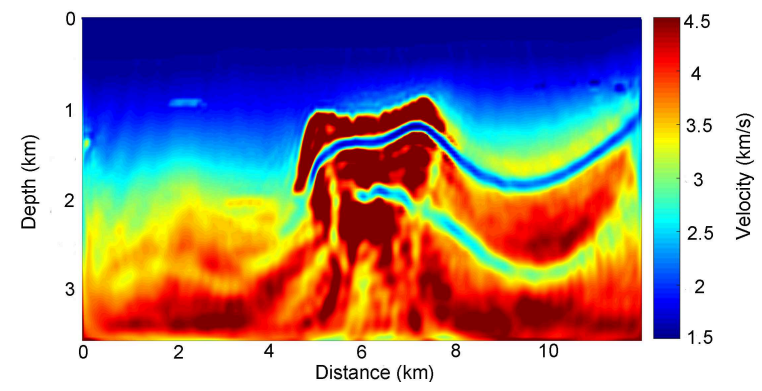

Fig. 24. The inverted BP salt model using WRI.

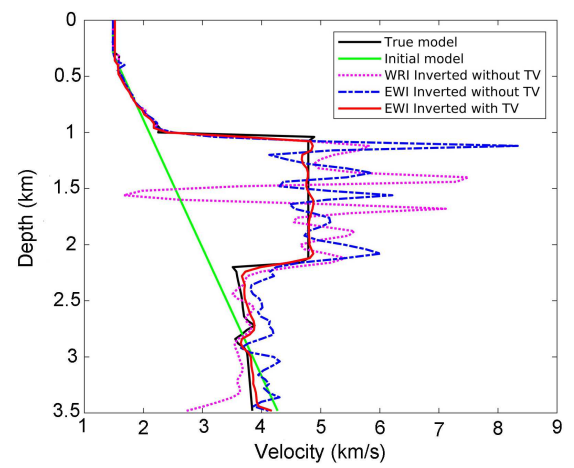

Fig. 25. The velocity profile at $6 \mathrm{~km}$.

Continental Shelf [45]. Due to the low signal-to-noise ratio (S/R), frequencies below $2.5 \mathrm{~Hz}$ has been filtered out. We show one shot gather in Fig. 26. There are 1824 shot gathers in the original data set at an interval of $0.01875 \mathrm{~km}$, and we choose 100 shot gathers to cover the 2D survey area. The target model we invert for is $12.5 \mathrm{~km}$ long and $3.75 \mathrm{~km}$ in depth, and the initial velocity model and the estimated wavelet is borrowed from [46], [47], as shown in Figs. 27 and 28, respectively. We resample the data and use 324 receivers from each streamer with an interval of $25 \mathrm{~m}$.

We perform EWI in two stages in a hierarchal approach. The frequency spectrum of the two selected bands for inversion is shown in Fig. 29. In sage 1, we invert for the selected frequency band from $3 \mathrm{~Hz}$ to $6 \mathrm{~Hz}$ with a dense sampling of $0.15 \mathrm{~Hz}$. In sage 2, the selected frequency band is from $9 \mathrm{~Hz}$ to

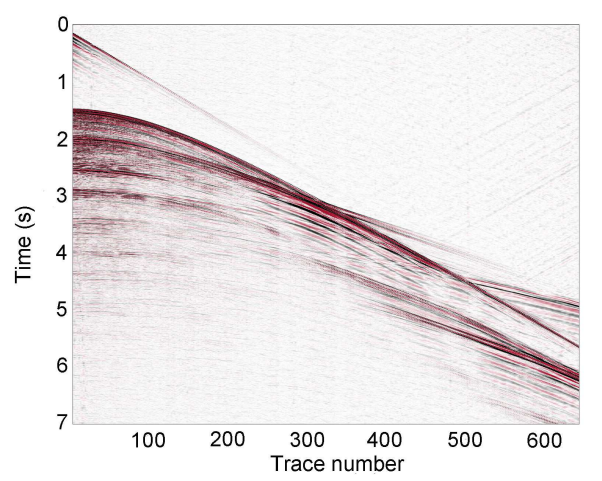

Fig. 26. One shot gather of the real dataset. 


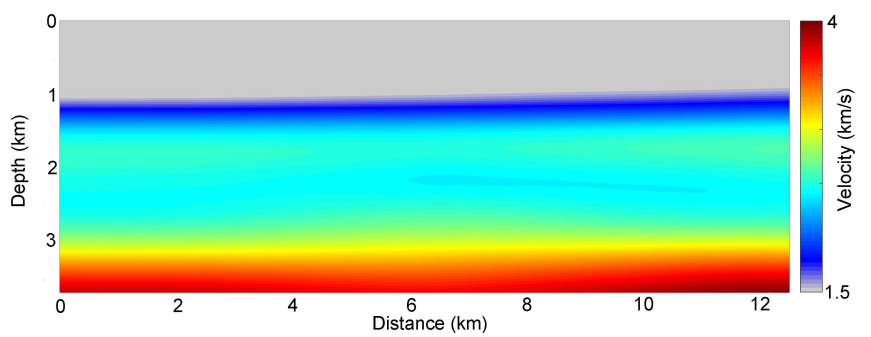

Fig. 27. The initial velocity model.

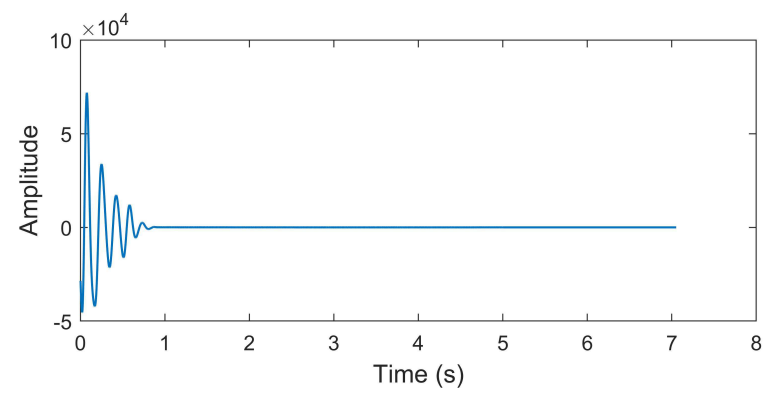

Fig. 28. The estimated wavelet.

$12 \mathrm{~Hz}$. We use two outer iterations, and two inner iterations in each frequency. The final inversion result is shown in Fig. 30. It is obvious that more geological information is recovered in the velocity model, especially the low velocity layers between $2.0 \mathrm{~km}$ to $2.5 \mathrm{~km}$ depth. The vertical velocity profile at 10.5 $\mathrm{km}$ is shown in Fig. 31, and the velocity between $1 \mathrm{~km}$ and $2 \mathrm{~km}$ is obtained by interpolation. It shows that the inverted velocity model from EWI with two outer iterations fits well$\log$ velocity reasonably well in the high velocity layers at the depths of $2.2 \mathrm{~km}$ and $3.2 \mathrm{~km}$, and also the low velocity layer at the depth of $3.4 \mathrm{~km}$. The background velocity between the depths of $2.2 \mathrm{~km}$ and $3.2 \mathrm{~km}$ gets averaged up. This may be caused by the unknown anisotropy in the background model, which is not taken into account in our isotropic inversion [48], [49]. In addition, we perform reverse time migration (RTM) using the initial and final velocities, and the images are shown in Figs. 32a and 32b, respectively. We realize improvements in the RTM image in multiple areas (as the red arrows point out) using the EWI inverted velocity. Finally, we compute the angle domain common-image gathers (ADCIGs) at locations $x=$ $\{1,2,3,4,5,6,7,8,9\} \mathrm{km}$ ranging between 0 to $40^{\circ}$ obtained using the space-shift imaging condition [50]. We note that the ADCIGs corresponding to the inverted velocity are flattened in multiple places compared to those corresponding to the initial velocity, which suggests an improvement in the kinematics of the inverted velocity.

\section{Discussion}

The key idea of EWI is to eliminate the dependency of the wavefield on the velocity using two independent parameters: the wavefield $u$ and a modified source function $f_{e}$. Initially, the reconstructed wavefield only contains single scattering components. Inner iterations add the multiscattering components to the wavefield with the help of the secondary sources (velocity

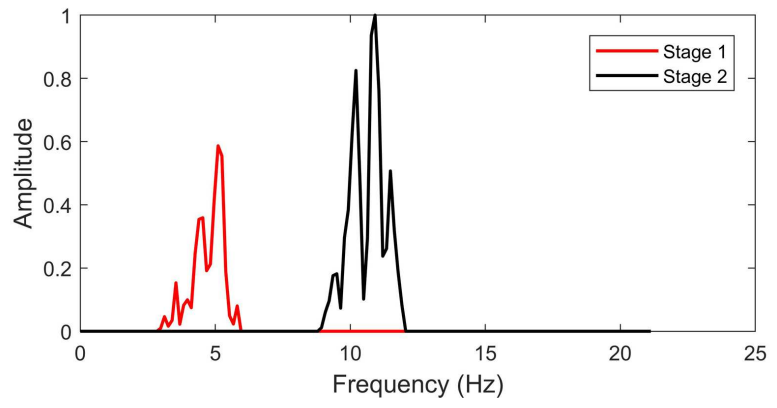

Fig. 29. The frequency spectrum of the selected frequency bands for stage 1 and stage 2 inversion.

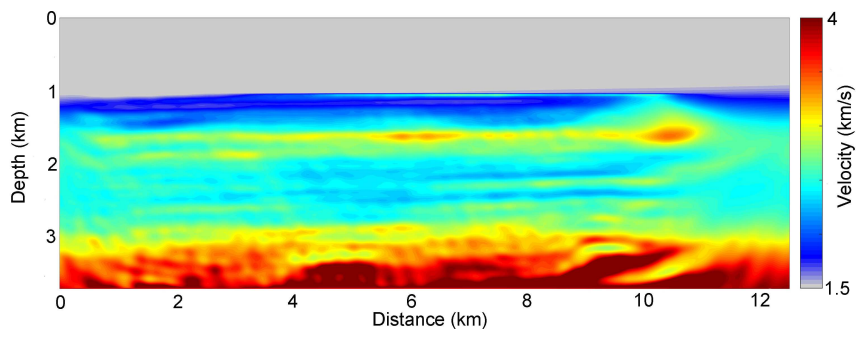

Fig. 30. The inverted velocity model.

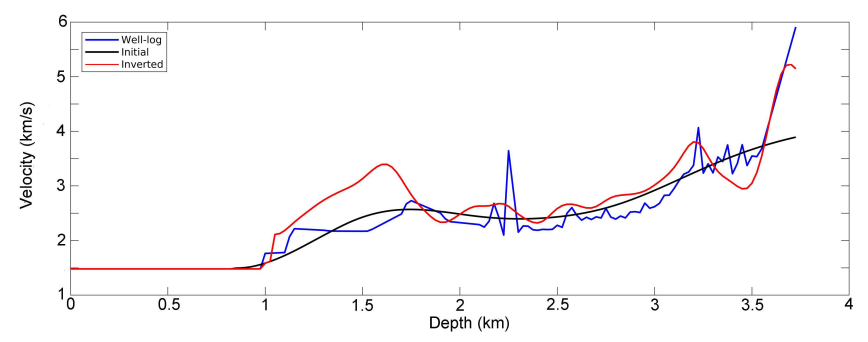

Fig. 31. The vertical velocity profile at $10.5 \mathrm{~km}$.
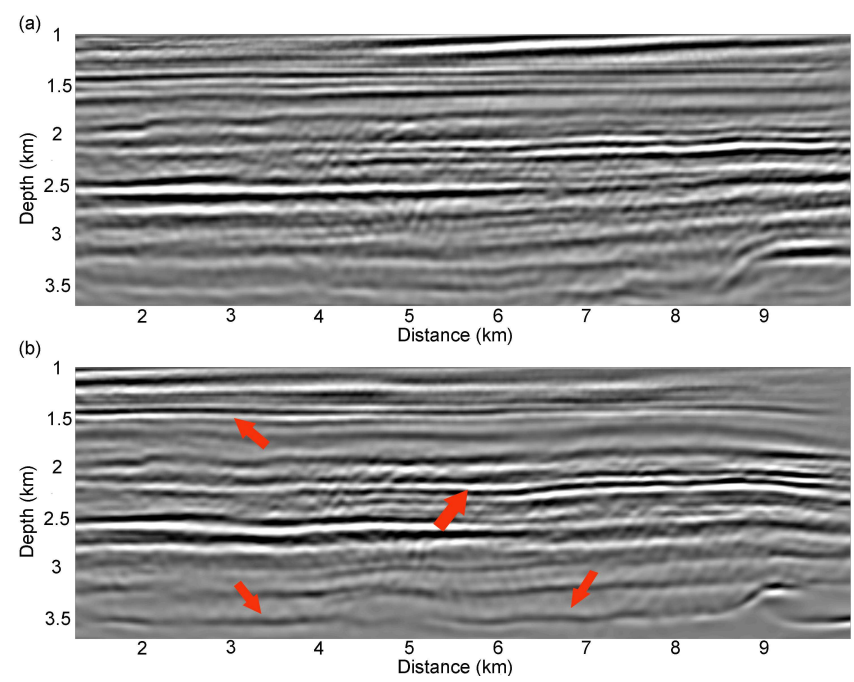

Fig. 32. The RTM images corresponding to the initial (a), and the final velocity (b). 


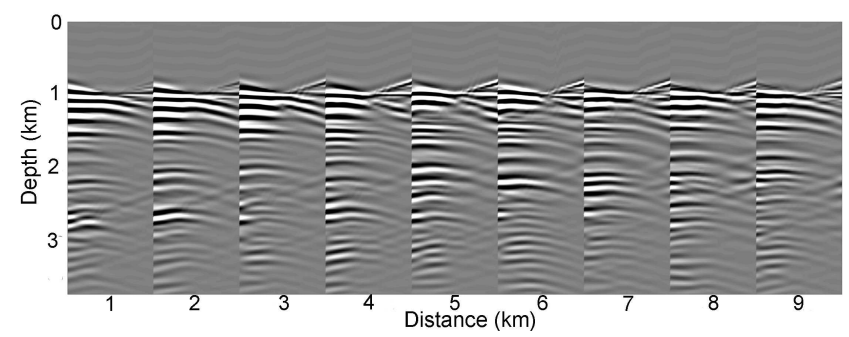

Fig. 33. ADCIGs at $x=\{1,2,3,4,5,6,7,8,9\} \mathrm{km}$ using the initial velocity.

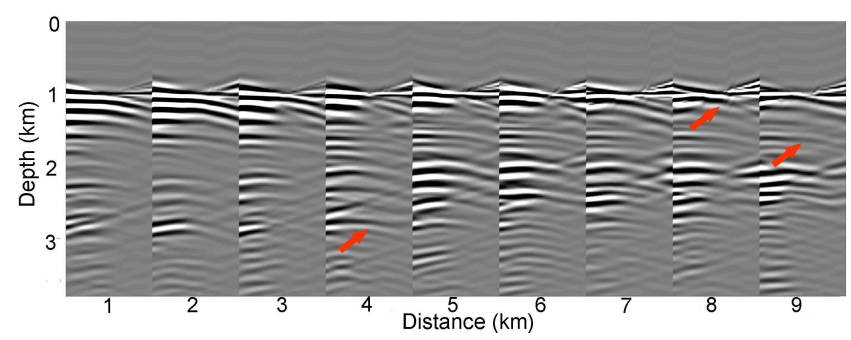

Fig. 34. ADCIGs at $x=\{1,2,3,4,5,6,7,8,9\} \mathrm{km}$ using the inverted velocity.

perturbations) included in $f_{e}$. In practise, two or three inner iterations is effective enough, as scattering energy higher than the second-order is reasonably weak to effectively contribute the velocity update. Although multiscattering components provide better illumination to the velocity model, the firstorder scattering is still the dominant component controlling the velocity update. As a result, if the velocity perturbation is large, we need to use outer iterations to improve the velocity inversion gradually. The velocity model is not part of the main optimization, so the TV constraint is applied to the follow up for inverting the velocity, unlike what is often done in TV regularized seismic waveform inversion [51], [34], [52]. As we update the velocity model, we apply the TV constraint to it as an independent step from the wavefied inversion. The TV was only needed for the salt body inversion.

As the modified source function $f_{e}$ is a dense vector, EWI requires twice the disk memory compared to WRI. As EWI doesn't need to calculate the background wavefields, it shares the same disk memory requirement with FWI. In frequencydomain waveform inversion, only a few discrete frequencies are needed, thus, the disk memory is not a big issue.

The weighting factor $\alpha^{2}$ controls how much the data fitting is imposed. For far away initial models, we should start with a relatively small $\alpha^{2}$ to enhance the data fitting. Several trial and error tests to find an appropriate $\alpha^{2}$ are recommended. However, when the initial velocity model is too far from the true one and low-frequency components are missing, even a small $\alpha^{2}$ and inner iterations fail to reconstruct a good wavefield. In this case, a number (usually few) outer iterations will help improve the inversion result.

\section{CONCLUSION}

We proposed outer iterations and a total variation constraint to improve the efficient wavefield inversion (EWI). EWI is more efficient than full-waveform inversion and wavefield reconstruction inversion as it can achieve convergence with only a few outer iterations. Besides the high efficiency, as EWI calculates the velocity perturbation through a direct division, the method reduces the cross-talk issue in multisource waveform inversion. With the help of outer iterations and total variation (TV) constraint, EWI can invert for a reasonably good velocity model even from relatively high frequencies with limited number of iterations. Applications to synthetic data generated from a modified Marmousi model and the BP salt model show that EWI generate reasonable inversion results at a low computational cost. A 2D field data also demonstrates the validity of the proposed approach.

\section{ACKNOWLEDGMENT}

We thank KAUST for its support and the SWAG group for the collaborative environment. This work utilized the resources of the Supercomputing Laboratory at King Abdullah University of Science and Technology (KAUST) in Thuwal, Saudi Arabia, and we are grateful for that. We also thank the associate editor and the reviewers for their helpful review and fruitful suggestions of the paper.

\section{REFERENCES}

[1] A. Tarantola, "Inversion of seismic reflection data in the acoustic approximation," Geophysics, vol. 49, no. 8, pp. 1259-1266, 1984.

[2] T. Alkhalifah, B. B. Sun, and Z. Wu, "Full model wavenumber inversion: Identifying sources of information for the elusive middle model wavenumbers," Geophysics, vol. 83, no. 6, pp. R597-R610, 2018.

[3] J. Virieux and S. Operto, "An overview of full-waveform inversion in exploration geophysics," Geophysics, vol. 74, no. 6, pp. WCC1-WCC26, 2009.

[4] F. Clement, G. Chavent, and S. G., "Migration-based traveltime waveform inversion of 2-d simple structures: A synthetic example," Geophysics, vol. 66, pp. 845-860, 2001.

[5] B. Biondi and A. Almomin, "Simultaneous inversion of full data bandwidth by tomographic full-waveform inversion," Geophysics, vol. 79, no. 3, pp. WA129-WA140, 2014.

[6] Y. Luo and G. T. Schuster, "Wave-equation traveltime inversion," Geophysics, vol. 56, no. 5, pp. 645-653, 1991.

[7] Y. Ma and D. Hale, "Wave-equation reflection traveltime inversion with dynamic warping and full-waveform inversion," Geophysics, vol. 78, no. 6, pp. R223-R233, 2013.

[8] B. Biondi and W. Symes, "Angle-domain common-image gathers for migration velocity analysis by wavefield-continuation imaging," Geophysics, vol. 69, pp. 1283-1298, 2004.

[9] W. W. Symes, "Migration velocity analysis and waveform inversion," Geophysical prospecting, vol. 56, pp. 765-790, 2008.

[10] C. Shin and Y. H. Cha, "Waveform inversion in the laplace domain," Geophysical Journal International, vol. 173, no. 3, pp. 922-931, 2008.

[11] R.-S. Wu, J. Luo, and B. Wu, "Seismic envelope inversion and modulation signal model," Geophysics, vol. 79, no. 3, pp. WA13-WA24, 2014.

[12] Z. Gao, Z. Pan, J. Gao, and R.-S. Wu, "Frequency controllable envelope operator and its application in multiscale full-waveform inversion," IEEE Transactions on Geoscience and Remote Sensing, vol. 57, no. 2, pp. 683-699, 2018.

[13] Y. Hu, R.-S. Wu, L.-G. Han, and P. Zhang, "Joint multiscale direct envelope inversion of phase and amplitude in the time-frequency domain," IEEE Transactions on Geoscience and Remote Sensing, 2019.

[14] S. Xu, D. Wang, F. Chen, Y. Zhang, and G. Lambare, "Full waveform inversion for reflected seismic data," pp. cp-293, 2012.

[15] H. Zhou, L. Amundsen, and G. Zhang, "Fundamental issues in full waveform inversion," pp. 1-5, 2012.

[16] T. Alkhalifah and Z. Wu, "The natural combination of full and imagebased waveform inversion," Geophysical Prospecting, vol. 64, no. 1, pp. 19-30, 2016. 
[17] Z. Wu and T. Alkhalifah, "Simultaneous inversion of the background velocity and the perturbation in full-waveform inversion," Geophysics, vol. 80, no. 6, pp. R317-R329, 2015.

[18] T. Alkhalifah, "Scattering-angle based filtering of the waveform inversion gradients," Geophysical Journal International, vol. 200, no. 1, pp. 363-373, 2014.

[19] Z. Wu and T. Alkhalifah, "Efficient scattering-angle enrichment for a nonlinear inversion of the background and perturbations components of a velocity model," Geophysical Journal International, vol. 210, no. 3, pp. 1981-1992, 2017.

[20] G. Wang, S. Yuan, and S. Wang, "Retrieving low-wavenumber information in fwi: An efficient solution for cycle skipping," IEEE Geoscience and Remote Sensing Letters, 2019.

[21] M. Warner and L. Guasch, "Adaptive waveform inversion: Theory," Geophysics, vol. 81, no. 6, pp. R429-R445, 2016.

[22] B. Sun and T. Alkhalifah, "A robust waveform inversion using a global comparison of modeled and observed data," The Leading Edge, vol. 38, no. 3, pp. 185-192, 2019.

[23] Y. Lin and L. Huang, "Acoustic-and elastic-waveform inversion using a modified total-variation regularization scheme," Geophysical Journal International, vol. 200, no. 1, pp. 489-502, 2014.

[24] B. Peters and F. J. Herrmann, "Constraints versus penalties for edgepreserving full-waveform inversion," The Leading Edge, vol. 36, no. 1, pp. 94-100, 2017.

[25] H. S. Aghamiry, A. Gholami, and S. Operto, "Compound regularization of full-waveform inversion for imaging piecewise media," IEEE Transactions on Geoscience and Remote Sensing, 2019.

[26] T. V. Leeuwen and F. Herrmann, "Mitigating local minima in fullwaveform inversion by expanding the search space," Geophysical Journal International, vol. 195, pp. 661-667, 2013.

[27] —, "A penalty method for pde-constrained optimization in inverse problems," Inverse Problems, vol. 32, p. 015007, 2015.

[28] A. Abubakar, W. Hu, T. Habashy, and M. P. B. van, "Application of the finite-difference contrast-source inversion algorithm to seismic fullwaveform data," Geophysics, vol. 74, no. 6, pp. WCC47-WCC58, 2009.

[29] C. Wang, D. Yingst, P. Farmer, I. Jones, G. Martin, and J. Leveille, "Fullwaveform inversion with the reconstructed wavefield method," SEG Technical Program Expanded Abstracts 2016, pp. 1237-1241, 2016.

[30] "Reconstructed full-waveform inversion with the extended source," SEG Technical Program Expanded Abstracts 2017, pp. 1449$1453,2017$.

[31] T. Alkhalifah, "Linear wavefield optimization using a modified source," Communications in computational physics, p. accepted, 2019.

[32] T. Alkhalifah and C. Song, "An efficient wavefield inversion," 81th EAGE Conference and Exhibition, 2019.

[33] E. Esser, L. Guasch, T. van Leeuwen, A. Y. Aravkin, and F. J. Herrmann, "Total variation regularization strategies in full-waveform inversion," SIAM Journal on Imaging Sciences, vol. 11, no. 1, pp. 376-406, 2018.

[34] M. Kalita, V. Kazei, Y. Choi, and T. Alkhalifah, "Regularized fullwaveform inversion with automated salt flooding," Geophysics, vol. 84, no. 4, pp. R569-R582, 2019.

[35] N. V. da Silva and G. Yao, "Wavefield reconstruction inversion with a multiplicative cost function," Inverse Problems, vol. 34, no. 1, p. 015004, 2017.

[36] L. Fu and W. W. Symes, "A discrepancy-based penalty method for extended waveform inversion," Geophysics, vol. 82, no. 5, pp. R287R298, 2017.

[37] T. Alkhalifah and C. Song, "An efficient wavefield inversion: Using a modified source function in the wave equation," Geophysics, vol. 84, no. 6, pp. R921-R934, 2019.

[38] R. A. Rutenbar, "Simulated annealing algorithms: An overview," IEEE Circuits and Devices magazine, vol. 5, no. 1, pp. 19-26, 1989.

[39] T. Alkhalifah and $\mathrm{Z}$. Wu, "Multiscattering inversion for low-model wavenumbers," Geophysics, vol. 81, no. 6, pp. R417-R428, 2016.

[40] C. Song, Z. Wu, and T. Alkhalifah, "Passive seismic event estimation using multiscattering waveform inversion," Geophysics, vol. 84, no. 3, pp. KS59-KS69, 2019.

[41] L. I. Rudin, S. Osher, and E. Fatemi, "Nonlinear total variation based noise removal algorithms," Physica D: nonlinear phenomena, vol. 60, no. 1-4, pp. 259-268, 1992.

[42] C. R. Vogel and M. E. Oman, "Iterative methods for total variation denoising," SIAM Journal on Scientific Computing, vol. 17, no. 1, pp. 227-238, 1996

[43] R. G. Pratt, "Seismic waveform inversion in the frequency domain, part 1: Theory and verification in a physical scale model," Geophysics, vol. 64, no. 3, pp. 888-901, 1999.
[44] C. Li, J.-P. Huang, Z.-C. Li, and R.-R. Wang, "Regularized least-squares migration of simultaneous-source seismic data with adaptive singular spectrum analysis," Petroleum science, vol. 14, no. 1, pp. 61-74, 2017.

[45] R. Soubaras and R. Dowle, "Variable-depth streamer-a broadband marine solution," first break, vol. 28, no. 12, 2010.

[46] M. Kalita and T. Alkhalifah, "Efficient full waveform inversion using the excitation representation of the source wavefield," Geophysical Journal International, vol. 210, no. 3, pp. 1581-1594, 2017.

[47] - "Flux-corrected transport for full-waveform inversion," Geophysical Journal International, vol. 217, no. 3, pp. 2147-2164, 2019.

[48] Z. Wu and T. Alkhalifah, "Waveform inversion for acoustic vti media in frequency domain," in SEG Technical Program Expanded Abstracts 2016. Society of Exploration Geophysicists, 2016, pp. 1184-1189.

[49] R. Djebbi and T. Alkhalifah, "Frequency domain multiparameter acoustic inversion for transversely isotropic media with a vertical axis of symmetry," Geophysics, vol. 84, no. 1, pp. C1-C14, 2018.

[50] P. C. Sava and S. Fomel, "Angle-domain common-image gathers by wavefield continuation methods," Geophysics, vol. 68, no. 3, pp. 10651074, 2003.

[51] P. Yong, W. Liao, J. Huang, and Z. Li, "Total variation regularization for seismic waveform inversion using an adaptive primal dual hybrid gradient method," Inverse Problems, vol. 34, no. 4, p. 045006, 2018.

[52] H. S. Aghamiry, A. Gholami, and S. Operto, "Implementing bound constraints and total-variation regularization in extended full-waveform inversion with the alternating direction method of multiplier: application to large contrast media," Geophysical Journal International, vol. 218, no. 2, pp. 855-872, 2019. 